\title{
Preparation of smart soft materials using molecular complexes
}

\author{
Takashi Miyata ${ }^{1,2,3}$
}

This article provides a short overview of our research regarding the preparation of smart soft materials using molecular complexes that reversibly associate and dissociate in response to environmental changes. Stimuli-responsive hydrogels that show swelling/shrinking in response to $\mathrm{pH}$ and temperature were prepared by copolymerization of a monomer bearing a phosphate group and other various monomers. Hydrogels with phosphate groups were useful tools for the construction of self-regulated drug delivery systems. Furthermore, we proposed a strategy for the preparation of biomolecule-responsive hydrogels that use reversible crosslinks in the networks of biomolecular complexes. Based on this strategy, we have prepared two types of biomolecule-responsive hydrogel that undergo changes in volume in response to target biomolecules. This was accomplished using biomolecular complexes such as antigen-antibody complexes and saccharide-lectin complexes and both a biomolecule-crosslinked hydrogel and a biomolecule-imprinted hydrogel have been synthesized with this approach. Biomolecule-crosslinked hydrogels, such as glucose- and antigen-responsive hydrogels, swelled in the presence of a target biomolecule due to the dissociation of biomolecular complexes that act as reversible crosslinks. On the other hand, biomolecule-imprinted hydrogels, such as tumor marker glycoprotein-responsive hydrogels, shrank in response to a target biomolecule due to the formation of a complex between ligands (lectin and antibody) and the target biomolecule. Thus, biomolecule-responsive hydrogels have many potential applications as smart biomaterials in biomedical fields. Although most smart soft materials prepared using molecular complexes still require further research, they are likely to become important materials in the future.

Polymer Journal (2010) 42, 277-289; doi:10.1038/pj.2010.12; published online 10 March 2010

Keywords: bioconjugation; complex; crosslink; hydrogel; molecular recognition; soft material; stimuli-responsive hydrogel

\section{INTRODUCTION}

Hydrogels are soft materials consisting of physically or chemically crosslinked polymer networks in aqueous solutions. Hydrogels show fascinating properties such as swelling, mechanical, permeation, optical and biocompatible properties that enable their use as adsorbents, chromatography columns, contact lenses, foods, industrial materials, etc. ${ }^{1-3}$ In addition, some hydrogels exhibit the unique property of undergoing an abrupt change in volume in response to environmental changes including $\mathrm{pH}$ and temperature. ${ }^{4-7}$ These unique hydrogels, called stimuli-responsive hydrogels, have attracted much attention as smart soft materials for mimicking natural feedback systems because they can sense a stimulus and induce a change in volume. Stimuli-responsive hydrogels have future applications in smart systems such as switches, sensors, actuators, bioreactors, drug delivery systems, cell culture systems and so on.

A variety of stimuli-responsive hydrogels that show reversible swelling/shrinking in response to environmental changes such as $\mathrm{pH}^{8,9}$ temperature, ${ }^{10-14}$ electric field ${ }^{15,16}$ and light ${ }^{17,18}$ have been prepared for the fabrication of self-regulated and controllable systems. For example, many researchers have focused on $\mathrm{pH}$ and temperatureresponsive hydrogels that can be prepared from poly(acrylic acid) (PAAc) and poly( $N$-isopropylacrylamide) (PNIPAAm), respectively, for applications in self-regulated drug delivery, because temperature and $\mathrm{pH}$ are the most widely used triggers for the monitoring of physiological changes by modulated smart systems. ${ }^{19-27}$ In addition, organs correspond to the presence of specific molecules as well as physicochemical changes such as $\mathrm{pH}$ and temperature. The natural feedback systems associated with biosystems to maintain life perceive specific ions or biological molecules such as hormones and elicit biological functions. To fabricate self-regulated systems by mimicking natural feedback systems, stimuli-responsive hydrogels that undergo structural changes in response to a specific biomolecule, that is, biomolecule-responsive hydrogels, are required. ${ }^{28-30}$ For example, glycoproteins have been used as tumor-specific markers for the monitoring of biological conditions of humans and biomoleculeresponsive hydrogels that can respond to glycoproteins are promising

${ }^{1}$ Department of Chemistry and Materials Engineering, Kansai University, 3-3-35 Yamate-cho, Suita, Osaka, Japan; 2PRESTO, Japan Science and Technology Agency (JST), 4-1-8 Honcho, Kawaguchi, Saitama, Japan and ${ }^{3}$ High Technology Research Center, Kansai University, Suita, Osaka, Japan

Correspondence: Professor T Miyata, Department of Chemistry and Materials Engineering, Kansai University, 3-3-35 Yamate-cho, Suita, Osaka 564-8680, Japan.

E-mail: tmiyata@kansai-u.ac.jp

Received 22 January 2010; revised 24 January 2010; accepted 26 January 2010; published online 10 March 2010 
smart biomaterials for the construction of molecular diagnostic systems and self-regulated drug delivery systems. A few researchers have reported glucose-responsive hydrogels for self-regulated insulin delivery systems in which a specific amount of insulin is administered in response to the concentration of glucose. ${ }^{31-40}$ In spite of their potential applications in biomedical fields, no studies on biomoleculeresponsive hydrogels responding to a specific biomolecule other than glucose have been undertaken. This is due to the difficulties in combining the ability to recognize a target biomolecule with the ability to cause a structural change in a hydrogel network.

We have proposed a novel strategy for the preparation of biomolecule-responsive hydrogels in which biomolecular complexes are used as reversible crosslinks in hydrogel networks. Based on our strategy, a variety of biomolecule-responsive hydrogels that undergo a change in volume in response to target biomolecules, such as monosaccharides and proteins, have been designed as smart soft materials. Furthermore, stimuli-responsive hydrogels responding to both $\mathrm{pH}$ and temperature were prepared by copolymerization of a monomer bearing a phosphate group and other various functional monomers. Thus, stimuliresponsive release of a protein drug from the hydrogels was achieved using complex formation between the phosphate group and the drug. The present paper provides an overview of our studies on a variety of smart soft materials using molecular complexes, including biomolecule-responsive hydrogels and other stimuli-responsive hydrogels. Our strategy for the design of various smart soft materials is described and focuses on the presence of functional groups and reversible crosslinks in hydrogel networks.

\section{STRATEGY FOR PREPARING SMART SOFT MATERIALS}

Biomolecules such as antibodies and lectins can recognize a target biomolecule and form a unique complex. Formation and dissociation of biomolecular complexes can be controlled by environmental changes. For example, lectins are carbohydrate-binding proteins that form complexes with specific saccharides and dissociate due to the presence of another saccharide. The saccharide acts as an inhibitor and the dissociation of the complex is based on the complex exchange mechanism. Thus, biomolecular complexes are sensitive to the presence and absence of a specific biomolecule. Owing to these properties, we have focused on a variety of biomolecular complexes for creating smart soft materials that respond to a target biomolecule and for fabricating smart drug delivery systems.

Drugs or probes that are positively or negatively charged interact with oppositely charged polymer networks. The resultant complexes dissociate and associate due to environmental changes such as $\mathrm{pH}$ and ionic strength. Therefore, the $\mathrm{pH}$-triggered release of a charged drug from an oppositely charged polymer network can be achieved using stimuli-responsive complex formation. Furthermore, the network structure of soft materials can be characterized by detecting fluorescence resonance energy transfer (FRET) caused by complex formation between the fluorescence donor and networks bearing the acceptor moiety.

We also proposed a strategy for the preparation of smart soft materials that recognize a target biomolecule and induce structural change. Our strategy for preparing such biomolecule-responsive hydrogels was to use biomolecular complexes as reversible crosslinks that dissociate and associate in the presence and absence of a target biomolecule, respectively. Based on this strategy, we have developed two types of biomolecule-responsive hydrogels that undergo a change in volume in response to an antigen and a glycoprotein that serves as a tumor marker. We constructed one system composed of a biomolecule-crosslinked hydrogel and another composed of a biomolecule-
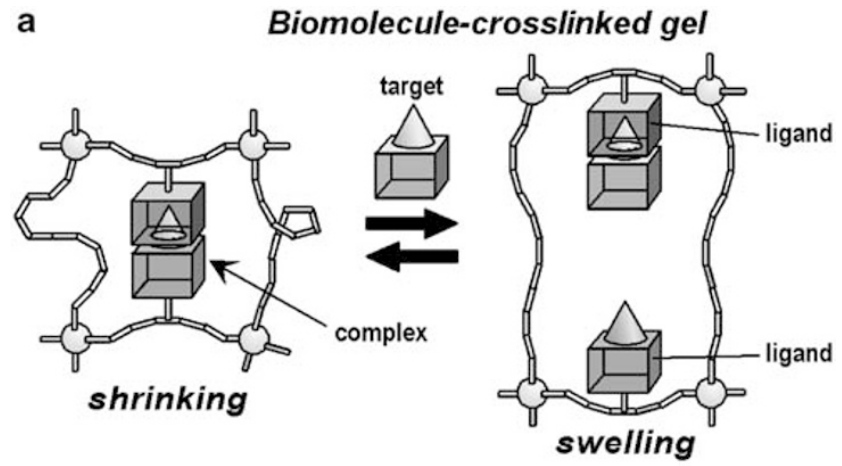

b

Biomolecule-imprinted gel
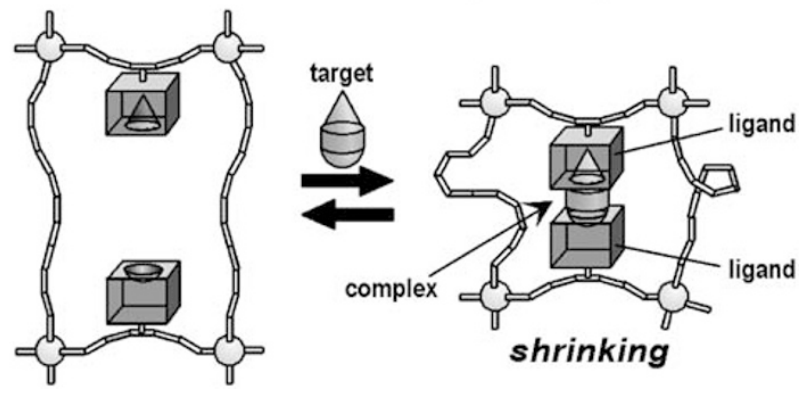

Figure 1 Schematic of swelling/shrinking behavior of biomolecule-responsive hydrogels: (a) biomolecule-crosslinked hydrogel; (b) biomolecule-imprinted hydrogel.

imprinted hydrogel (Figure 1). The biomolecule-crosslinked hydrogel swells in the presence of a target biomolecule because of a decrease in crosslinking density resulting from complex exchange. The biomolecule-imprinted hydrogel shrinks in response to a target biomolecule because the crosslinking density increases due to the formation of sandwich-like complexes that act as crosslinks. Thus, we have developed smart soft materials using molecular complexes that are sensitive to stimuli such as $\mathrm{pH}$ and the presence of biomolecules. The following sections will provide a short overview of our research into the synthesis of smart soft materials and the fabrication of smart systems.

\section{PREPARATION OF HYDROGELS RESPONDING TO BOTH PH AND TEMPERATURE}

Temperature and $\mathrm{pH}$-responsive hydrogels have attracted much attention as smart soft materials because temperature and $\mathrm{pH}$ are the most usual and controllable stimuli. Temperature-responsive hydrogels can be prepared from polymers that have a low critical solution temperature, such as PNIPAAm. The introduction of functional groups bearing electrolytes, such as carboxyl or sulfonic groups, into the polymer chain enables the resultant hydrogels to undergo abrupt volume changes in response to $\mathrm{pH}$. We focused on the divalent phosphate group as an electrolyte for the introduction of unique pH-responsiveness into a hydrogel. ${ }^{41} \mathrm{~A}$ monomer with a phosphate group (phosmer) was used for the synthesis of $\mathrm{pH}$-responsive hydrogels that undergo volume changes at two different pHs. Stimuliresponsive behavior of various hydrogels bearing phosphate groups was investigated with various functional comonomers. Furthermore, positively charged lysozyme, a model protein drug, was loaded within the $\mathrm{pH}$-responsive hydrogel by complex formation with the phosphate groups and the release of the lysozyme from the hydrogel was investigated as a function of $\mathrm{pH}^{42,43}$ 


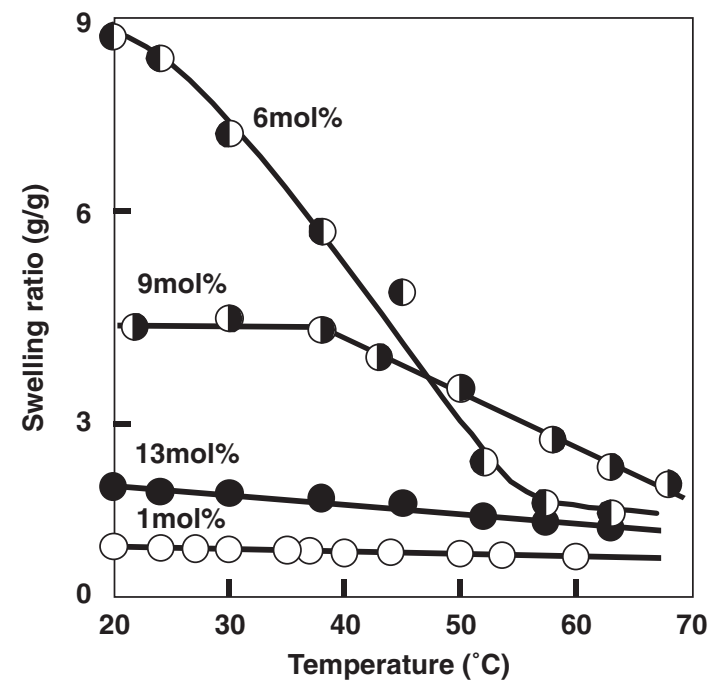

Figure 2 Temperature dependence of the swelling ratio for phosmer-HEMA hydrogels with a phosphate group content of $1,6,9$ and $13 \mathrm{~mol} \%$ in water.

First of all, phosmer was copolymerized with various monomers including 2-hydroxyethyl methacrylate (HEMA), acrylic acid (AAc) and $N, N^{\prime}$-dimethyl acrylamide for the preparation of $\mathrm{pH}$-responsive hydrogels bearing a variety of functional groups. The swelling ratio of the resultant copolymer hydrogels bearing phosphate groups was strongly dependent upon the hydrophobicity of the comonomer. Copolymer hydrogels bearing phosphate groups underwent volume changes at $\mathrm{pH} 5$ and 10 because the phosphate group was negatively charged when at these pHs. In addition, phosmer-HEMA copolymer hydrogels showed unique swelling/shrinking behavior in response to temperature. ${ }^{41}$ The phosmer-HEMA hydrogels with a phosphate group content of $6 \mathrm{~mol} \%$ swelled stepwise with increasing $\mathrm{pH}$ and shrank gradually with a rise in temperature (Figure 2). Thus, the phosmer-HEMA hydrogels were sensitive to both $\mathrm{pH}$ and temperature. The phosmer-HEMA hydrogels showed temperature-responsive swelling/shrinking behavior even though they were not composed of temperature-responsive polymer chains such as PNIPAAm. To clarify the mechanism of temperature-responsive behavior of phosmerHEMA hydrogels, a change in the polymer-solvent interaction parameter $(\chi)$ between hydrogel networks and water was examined as a function of temperature. An increase in $\chi$ with increasing temperature suggested that the temperature-responsive shrinking of the phosmerHEMA hydrogels was attributed to temperature dependent affinity of the phosmer-HEMA polymer chains with water. Therefore, the temperature-responsive behavior of the phosmer-HEMA hydrogels was directly influenced by the composition of the copolymer.

Positively charged drugs can be loaded within hydrogels that contain negatively charged groups by complex formation based on electrostatic interactions. ${ }^{44,45}$ We prepared temperature- and $\mathrm{pH}$ responsive hydrogels by copolymerization of phosmer with NIPAAm, and loaded lysozyme, a positively charged protein, into the hydrogel by complex formation. ${ }^{42,43}$ The amount of lysozyme loaded into phosmer-NIPAAm hydrogels was compared with phosmer-AAc hydrogels that had carboxyl groups. Lysozyme was more effectively loaded into phosmer-NIPAAm hydrogels than into phosmer-AAc hydrogels. This indicates that the phosphate group is more effective in complexing positively charged drugs than the carboxyl group.

Lysozyme release experiments from the phosmer-NIPAAm hydrogels were carried out as a function of $\mathrm{pH}$ because complex formation

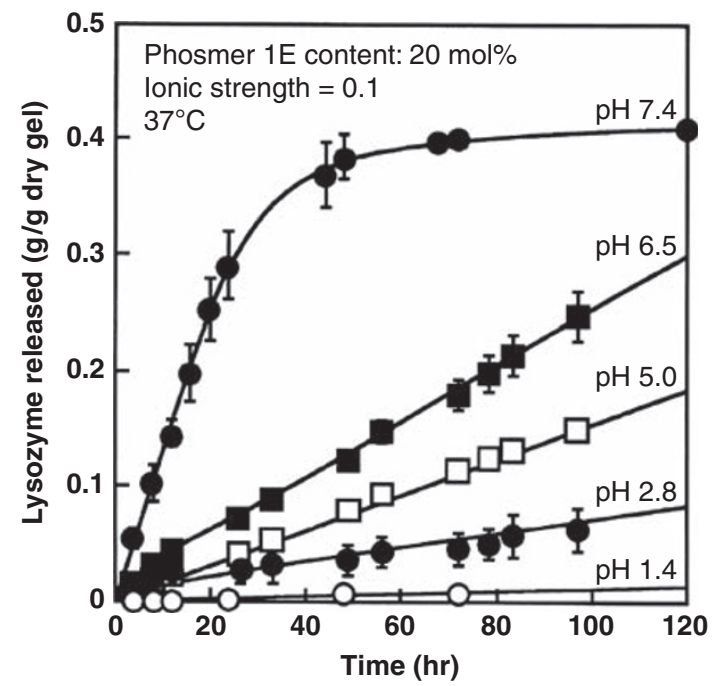

Figure 3 Amount of lysozyme released from the phosmer-NIPAAm hydrogel at different $\mathrm{pH}$ values.

between negatively and positively charged groups is strongly influenced by the $\mathrm{pH}$. Lysozyme was abruptly released from the phosmerNIPAAm hydrogel into the external solution at $\mathrm{pH} 7.4$ but was not released at $\mathrm{pH} 1.4$ (Figure 3 ). The lysozyme release profile matched the swelling behavior of hydrogels and was associated with osmotic expansion of the ionic network. As a result, a $\mathrm{pH}$-responsive ONOFF release of lysozyme was achieved by complex formation and the dissociation of phosphate groups and lysozyme. This $\mathrm{pH}$-responsive ON-OFF drug release revealed that the phosmer-NIPAAm hydrogel is a promising smart biomaterial for intestine-targeted protein delivery. This can be achieved by oral administration of the protein drug and retention within the hydrogel under the acidic conditions of the stomach. Rapid release occurs upon contact with the less acidic intestinal environment.

\section{GLUCOSE-RESPONSIVE HYDROGELS}

Insulin is a hormone that controls glucose metabolism and is secreted from the Wrangell Hans Island of the pancreas. The inability of the pancreas to control blood glucose concentration causes diabetes. Therefore, in the treatment of diabetes, blood glucose levels are monitored and a specific amount of insulin is administered. Glucose-responsive hydrogels are smart soft materials that can act as an artificial pancreas to administer a necessary amount of insulin in response to blood glucose concentration. A few glucose-responsive hydrogels have been prepared for the development of self-regulated insulin release systems. The most general method for the development of self-regulated insulin release systems that respond to blood glucose concentration is the combination of glucose oxidase with polyelectrolyte hydrogels that display $\mathrm{pH}$-responsive swelling/shrinking behavior. $^{31-36}$ Glucose-responsive hydrogels that regulate insulin release have been prepared by loading glucose oxidase into $\mathrm{pH}$-responsive networks. In addition, totally synthetic hydrogels showing glucoseresponsive volume changes were prepared by the combination of temperature-responsive PNIPAAm with phenylboronic acids that form a complex with glucose. ${ }^{37-40}$ Temperature-responsive hydrogels with phenylboronic acids underwent abrupt volume changes in response to the concentration of glucose due to the formation of a complex between phenylboronic acid and glucose, which induced an low critical solution temperature shift of the hydrogel networks. Based 
on our strategy for preparing biomolecule-responsive hydrogels, we have prepared glucose-responsive hydrogels using biomolecular complexes as reversible crosslinks of the polymer network, which is distinct from previous methods.

Lectins are carbohydrate-binding proteins that form complexes with the carbohydrate chains of glycoproteins and glycolipids on the cell surface. Lectins have been used for fabricating sensing systems due to their unique properties of carbohydrate recognition ${ }^{46-51}$ and have been employed in the design of glucose-responsive systems that recognize glucose. For example, saccharide-responsive hydrogels were prepared by combining the carbohydrate-binding properties of concanavalin A (Con.A), which is a lectin that recognizes glucose and mannose, with the temperature-responsive property of PNIPAAm. ${ }^{52}$ We synthesized a variety of carbohydrate polymers by copolymerizing a monomer with pendant glucose (2-glucosyloxyethyl methacrylate: GEMA) and hydrophilic or hydrophobic monomers and examined their unique surface properties under various conditions. ${ }^{53-58}$ Furthermore, complex formation between Con.A and polymers with pendant glucose (PGEMA) was investigated to design a glucose-responsive polymer. ${ }^{59}$ When Con.A was added to a buffer solution containing PGEMA, the solution became turbid due to multiple associations between PGEMA and Con.A. The turbid solution containing PGEMA-Con.A complexes became transparent again by the addition of free glucose and mannose, but the addition of galactose did not make the solution transparent. These results indicate that Con.A preferentially forms a complex with free glucose or mannose rather than with pendant glucose of PGEMA and does not form a complex with galactose. We concluded that the complex of PGEMA and Con.A shows glucose- or mannose-responsive behavior. The monosaccharide-responsive behavior of the PGEMA-Con.A complex is useful in creating glucose-responsive hydrogels.

We developed glucose-responsive hydrogels that underwent volume changes in response to glucose concentration using complexes of PGEMA and Con.A as reversible crosslinks in hydrogel networks. ${ }^{60,61}$ The bioconjugated hydrogel in which Con.A was entrapped consisted of a PGEMA network (Con.A-entrapped PGEMA hydrogel) prepared by the copolymerization of GEMA and $N, N^{\prime}$-methylenebisacrylamide (MBAA) after the formation of the PGEMA-Con.A complex that acted as a reversible crosslink of the resultant network. ${ }^{60}$ When the resulting Con.A-entrapped PGEMA hydrogel and PGEMA hydrogel without Con.A were immersed into a buffer solution containing free glucose, the former swelled immediately but the volume of the latter did not change. Compressive modulus measurements revealed that the crosslinking density of the Con.A-entrapped PGEMA hydrogel decreased with increasing glucose concentration. Furthermore, the swelling ratio of Con.A-entrapped PGEMA hydrogel increased remarkably in the presence of mannose compared with glucose but did not change in the presence of galactose (Figure 4). The glucose- or mannose-responsive swelling behavior of Con.A-entrapped PGEMA hydrogels was attributed to the dissociation of PGEMA-Con.A complexes that provided reversible crosslinks (Figure 5). Con.A has a stronger affinity for mannose than glucose and has no affinity for galactose. Therefore, free mannose and glucose induced the dissociation of the PGEMA-Con.A complex by competitive complex exchange but free galactose did not. As a result, the Con.A-entrapped PGEMA hydrogels swelled in response to mannose and glucose due to decreasing crosslinking density but no change was observed in the presence of galactose. Thus, the Con.A-entrapped PGEMA hydrogels can recognize a specific monosaccharide and a structural change is induced.

To obtain glucose-responsive hydrogels that reversibly swell in the presence of free glucose and shrink in its absence, Con.A was

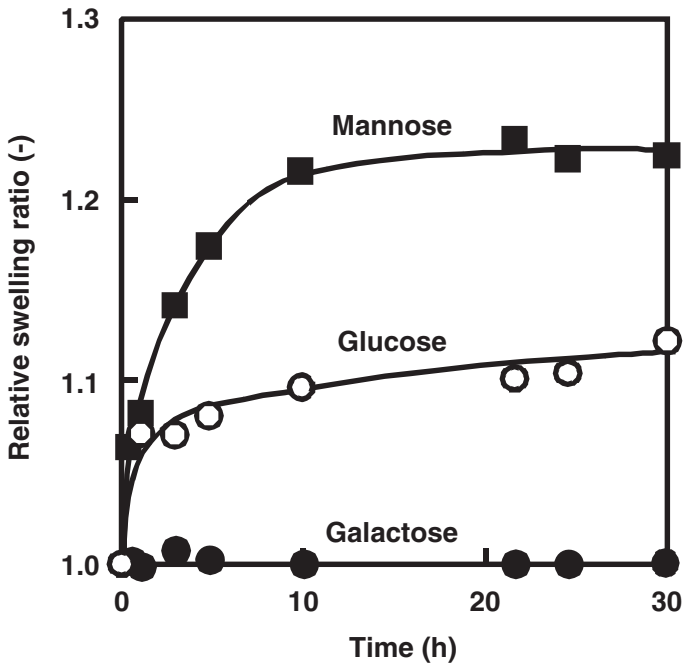

Figure 4 Change in the swelling ratio of Con.A-entrapped PGEMA hydrogels as a function of time when the hydrogel was immersed in $0.1 \mathrm{M}$ Tris- $\mathrm{HCl}$ buffer ( $\mathrm{pH}$ 7.5) containing 1 wt \% monosaccharide: $\bigcirc$, glucose; mannose; $\bullet$ galactose. The concentration of Con.A entrapped in the hydrogel is 18.8 wt \%.

covalently immobilized within the hydrogel. This soft material was synthesized by the copolymerization of GEMA with chemically modified Con.A bearing polymerizable groups. ${ }^{61}$ The Con.A-copolymerized PGEMA hydrogels showed reversible swelling/shrinking in response to stepwise changes in glucose concentration but the Con.Aentrapped hydrogel did not. Con.A leaks out of the Con.A-entrapped hydrogel during swelling in the presence of free glucose so the hydrogel cannot shrink in the absence of glucose. The Con.A-copolymerized hydrogel can undergo reversible volume change in response to glucose because Con.A was covalently immobilized within the hydrogel network and does not leak out of the hydrogel. Thus, covalent immobilization of Con.A within the PGEMA network enables the hydrogel to show reversible glucose-responsive swelling/shrinking behavior. The unique glucose-responsiveness of Con.A-copolymerized PGEMA hydrogels indicate that they are promising candidates as smart biomaterials for the fabrication of self-regulated insulin release systems, in which insulin can be administered in response to blood glucose concentration.

\section{ANTIGEN-RESPONSIVE HYDROGELS}

Because smart soft materials monitor physiological changes or guide drugs to a specific site, some researchers have developed proteinresponsive hydrogels that undergo structural changes triggered by selective enzyme catalysis. ${ }^{62-71}$ However, the structural changes of protein-responsive hydrogels in the literature were based on the degradation of polymer networks caused by an enzymatic reaction. Studies on protein-responsive hydrogels that undergo reversible swelling/shrinking in response to a target protein have not been undertaken in spite of their potential applications as smart biomaterials. This is due to the inherent difficulty in combining a mechanism for protein recognition with a mechanism for reversible swelling/shrinking of a hydrogel network.

An antibody recognizes a specific antigen and forms antigenantibody binding through multiple noncovalent bonds, including electrostatic interactions, hydrogen bonds and hydrophobic interactions. The specificity and versatility of antibodies provide useful tools 


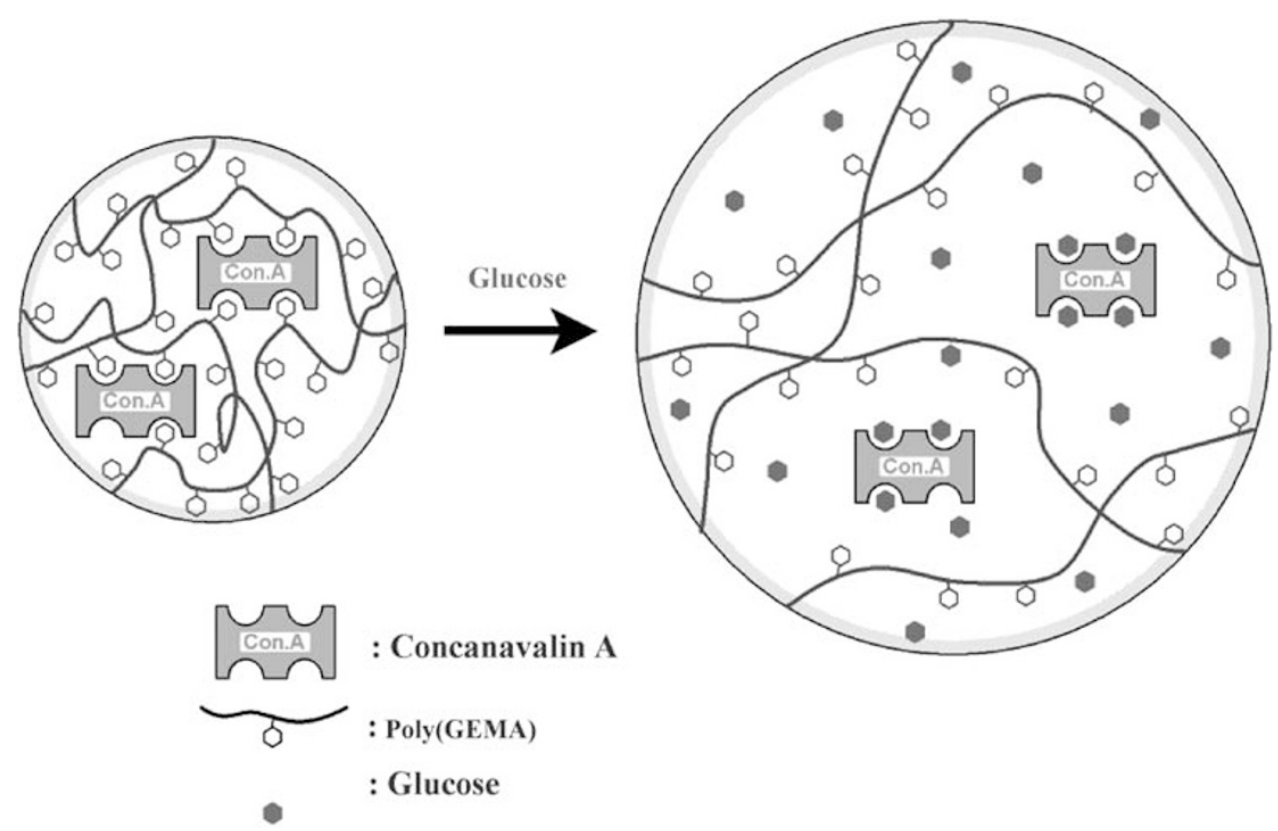

Figure 5 Schematic of glucose-responsive swelling behavior of Con.A-entrapped PGEMA hydrogels.

for the development of immunological assays and for the detection of signal biomolecules showing physiological changes. ${ }^{72}$ Using the antigen-binding properties of antibodies, we have developed antigenresponsive hydrogels that recognize a target antigen and induce a change in volume. ${ }^{73-75}$ Our approach for the preparation of antigenresponsive hydrogels was to use antigen-antibody bindings as reversible cross-links that form and dissociate in response to a target antigen. In our studies, rabbit IgG was used as an antigen and goat anti-rabbit IgG (GAR IgG) was used as an antibody. The aforementioned antibody and antigen formed an antigen-antibody binding that acted as a reversible cross-link of the hydrogel network. Antigenantibody entrapment hydrogels, in which an antibody acted as a crosslinker and formed an antigen-antibody binding with two antigens, were prepared by the copolymerization of rabbit IgG bearing polymerizable groups with acrylamide (AAm) and $N, N^{\prime}$-methylenebisacrylamide (MBAA) in the presence of GAR IgG. This polymerization was conducted after rabbit IgG bearing polymerizable groups formed an antigen-antibody binding with GAR IgG. ${ }^{74}$ The antigen-antibody entrapment hydrogels swelled gradually in a buffer solution containing rabbit IgG and the swelling ratio was strongly dependent upon the concentration of antigen in the buffer solution. These results indicate that antigen-antibody entrapment hydrogels are sensitive to a target antigen.

In general, potential applications of stimuli-responsive hydrogels require a reversible change in volume in response to changes in environmental stimuli. By forming a semi-interpenetrating polymer network (semi-IPN) composed of linear poly(acrylamide) (PAAm) with grafted antibodies (GAR IgG) and PAAm networks with grafted antigen (rabbit IgG), we designed a reversible antigen-responsive hydrogel that underwent reversible swelling/shrinking in response to a target antigen, in which complexes between grafted antibodies and grafted antigens acted as reversible crosslinks (Figure 6). ${ }^{73}$ The antigen-antibody semi-IPN hydrogels showed a gradual increase in the swelling ratio in the presence of rabbit IgG. However, the swelling ratio of hydrogel did not change in the presence of other IgGs (Figure 7). When the antigen-antibody semi-IPN hydrogel was alternately immersed into a buffer solution with and without rabbit IgG, it swelled immediately in the presence of rabbit IgG and shrank in its absence (Figure 8). However, the antigen-antibody entrapment hydrogel did not show reversible swelling/shrinking in response to stepwise changes in the concentration of antigen. These results indicate that an antigen-responsive hydrogel showing a reversible volume change can be prepared by the introduction of a semi-IPN structure. The reversible antigen-responsive volume change observed in the antigen-antibody semi-IPN hydrogels is attributed to reversible changes in cross-linking density, which results from the formation and dissociation of the bond between grafted antigen and grafted antibody in the absence and presence of a target antigen, respectively (Figure $6 \mathrm{~b}$ ). These results suggest that antigen-antibody semi-IPN hydrogels can recognize only rabbit IgG and induce reversible volume changes. Thus, these hydrogels are reversible antigenresponsive hydrogels.

We also investigated the effect of preparation conditions on antigen-responsive swelling of antigen-antibody entrapment hydrogels. ${ }^{75}$ To prepare antigen-antibody entrapment hydrogels showing significant antigen-responsive swelling behavior, modified antigens bearing only a vinyl group as a comonomer were used and found to be more effective than antigens possessing multiple vinyl groups. The number of vinyl groups in the modified antigen affected antigen-responsive swelling behavior of the resultant antigen-antibody entrapment hydrogels. The antigen bearing more than two vinyl groups acted as an irreversible covalent crosslink rather than a reversible crosslink. Furthermore, antigen-antibody entrapment hydrogels with a larger number of antigen-antibody bonds showed improved antigen-responsive swelling behavior. Thus, antigen-responsive hydrogels must be designed by controlling the preparation conditions and resultant network structures.

Biomolecule-responsive hydrogels are useful tools for the construction of self-regulated drug delivery systems where drugs are administered in response to physiological changes. To investigate the use of reversible antigen-responsive hydrogels as smart devices for selfregulated drug delivery, drug permeation experiments were conducted 
a

1) Introduction of vinyl groups into antigen and antibody

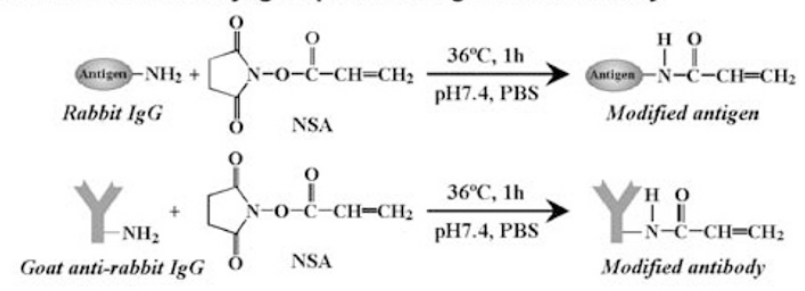

2) Coplymerization of vinyl-antigen and acrylamide

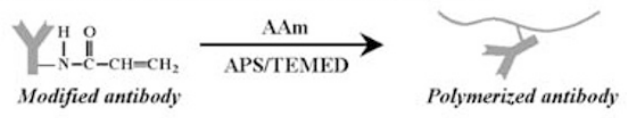

3) Preparation of a antigen-antibody semi-IPN gel

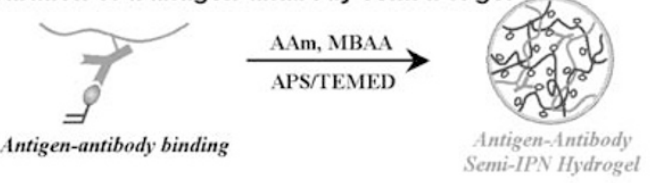

b
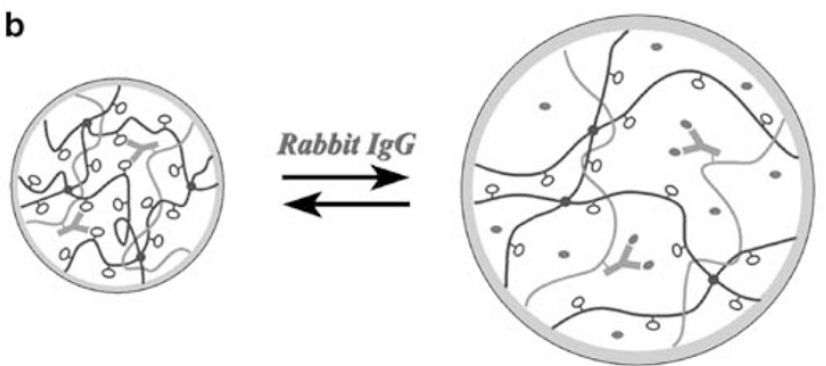

: Antibody-immobilized polymer chain

- : Free antigen (Rabbit IgG)

\% : Antigen-immobilized polymer chain

Figure 6 Strategy for the preparation of an antigen-responsive hydrogel. (a) Synthesis of the antigen-antibody semi-IPN hydrogel. (b) Tentative schema for the swelling of an antigen-antibody semi-IPN hydrogel in response to the target antigen.

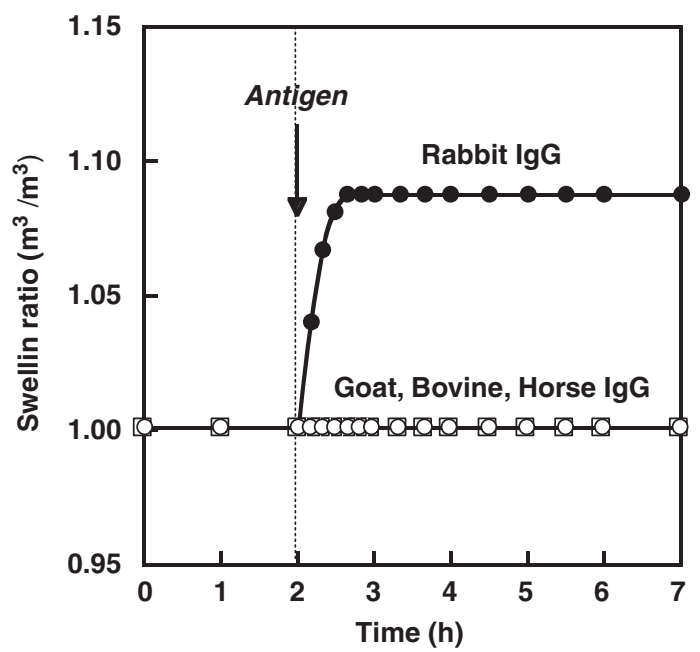

Figure 7 Changes in the swelling ratio of antigen-antibody semi-IPN hydrogels following the addition of rabbit IgG $(\bullet)$, goat IgG $(O)$, bovine IgG $(\square)$ and horse IgG ( $\boldsymbol{\square})$, after swelling had attained equilibrium in phosphate buffer solution. The concentration of the antigen in the phosphate buffer solution was $4 \mathrm{mg} \mathrm{ml}^{-1}$.

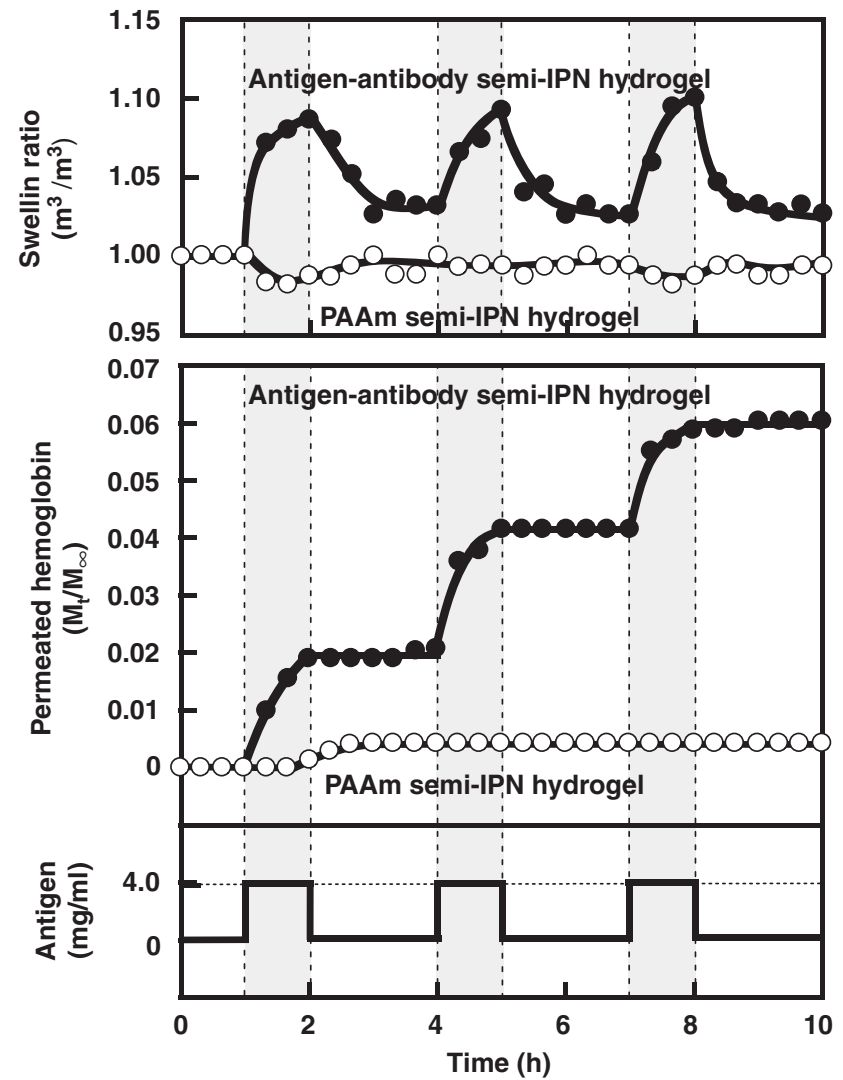

Figure 8 Changes in the swelling ratio of PAAm semi-IPN hydrogels $(O)$, antigen-antibody semi-IPN hydrogels $(\bullet)$ and the permeation profile of a model drug in response to stepwise changes in the concentration of antigen between 0 and $4 \mathrm{mg} \mathrm{ml}^{-1}$ at $25^{\circ} \mathrm{C}$.

using antigen-antibody semi-IPN and entrapment hydrogels. As a result, a drug permeated through the antigen-antibody semi-IPN hydrogel in the presence of a target antigen but not in its absence (Figure 8$).{ }^{73}$ The antigen-antibody semi-IPN hydrogels can control drug permeation in response to stepwise changes in the concentration of target antigen. On the other hand, ON-OFF drug release in response to antigen concentration was not achieved using the antigen-antibody entrapment hydrogel because the hydrogel showed an irreversible change in volume. Thus, antigen-antibody semi-IPN hydrogels showing reversible antigen-responsive swelling/shrinking are promising candidates for the fabrication of smart devices that modulate drug release in response to an antigen that shows physiological changes.

\section{BIOMOLECULE-IMPRINTED HYDROGELS}

Proteins such as enzymes, lectin and antibodies can recognize specific proteins and saccharides based on the fit of the guest molecule into their molecular cavities. Molecular imprinting has attracted much attention as a convenient technique for the creation of biomimetic polymers displaying molecular cavities for molecular recognition. ${ }^{76-82}$ In molecular imprinting, after functional monomers are prearranged around a print molecule by noncovalent interactions, the monomers are copolymerized with crosslinkers to form networks. The print molecule is then removed from the resultant polymer network to create a molecular cavity which is used as a molecular recognition site. A molecularly imprinted polymer possessing a molecular cavity can 
recognize the guest molecule (print molecule) on the basis of a combination of reversible binding and shape recognition. Most molecular imprinting requires a large amount of crosslinker for fixation of functional groups in the molecular recognition site. Recently, a few researchers have focused on molecular imprinting for the introduction of molecular recognition sites into temperatureresponsive hydrogels. ${ }^{83,84}$ Their studies revealed that molecular imprinting enables polymer networks to memorize a target molecule.

In general, functional monomers used in molecular imprinting are low molecular weight monomers bearing a functional group that acts as a ligand for a print molecule. However, we proposed a novel biomolecular imprinting technique in which biomolecules such as lectins and antibodies are used as ligands for a print biomolecule (Figure 9) ${ }^{85}$ Molecularly imprinted hydrogels can be prepared using a minute amount of crosslinker and the networks can memorize a target by using a biomolecule with molecular recognition functions as ligands. In addition, a hydrogel network with a minute amount of crosslinker can undergo a change in volume due to an increase in crosslinking density upon complex formation between ligand biomolecules and target biomolecules. Our strategy for the preparation of biomolecule-responsive hydrogels that shrink in response to a target biomolecule included biomolecules such as lectin and antibody, which

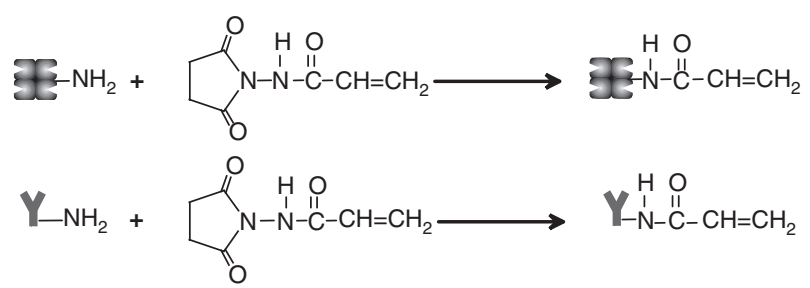

(ii) Preparation of AFP-imprinted gel
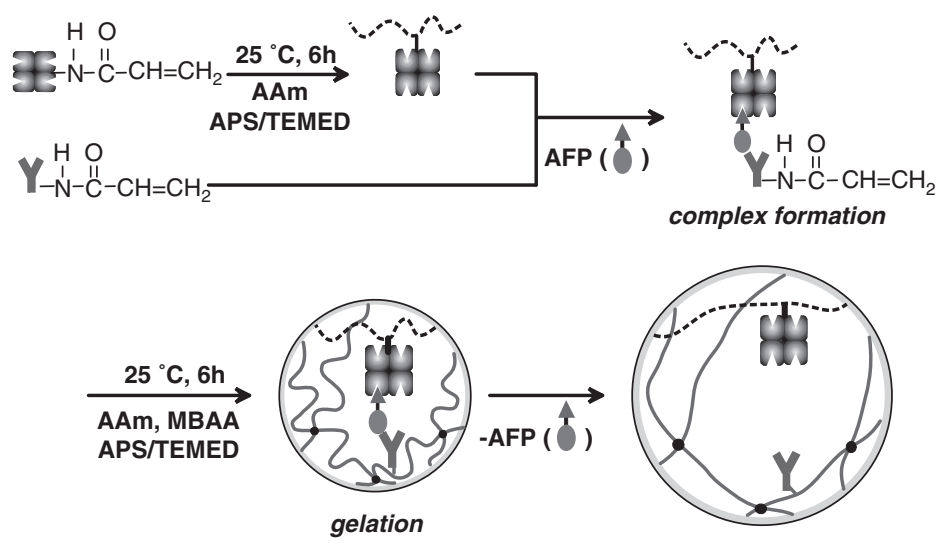

b

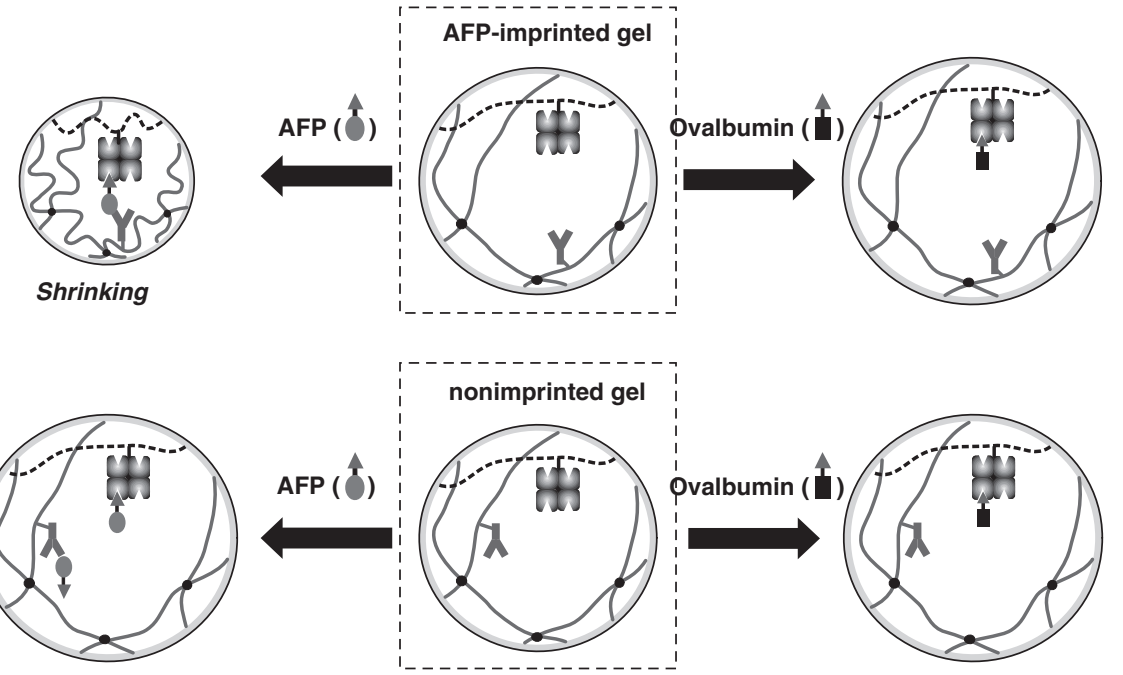

Figure 9 Strategy for the preparation of tumor-marker-responsive hydrogels by biomolecular imprinting: (a) Synthesis of the tumor marker-imprinted hydrogel using lectins and antibodies as ligands for template glycoprotein molecules (tumor-specific marker AFP). (b) A schematic representation of glycoproteinresponsive behavior of tumor marker-imprinted and non-imprinted hydrogels. 
are used as ligands for a print biomolecule. Using minute amounts of crosslinker in molecular imprinting enables the resultant hydrogels to undergo a change in volume in response to a target biomolecule. Based on this strategy, we prepared biomolecule-responsive hydrogels that induce structural changes in response to a target glycoprotein, using biomolecular imprinting.

$\alpha$-Fetoprotein (AFP) is a tumor-specific marker glycoprotein widely used for the diagnosis of primary hepatoma from serum. ${ }^{86}$ To prepare tumor marker-responsive hydrogels, we imprinted AFP in the hydrogel network by a biomolecular imprinting technique, in which lectins and antibodies were used as ligands for saccharide and peptide chains of AFP, respectively (Figure 9a). ${ }^{85}$ After PAAm-grafted lectins and acryloyl antibodies were synthesized, AAm was copolymerized with acryloyl-antibody and MBAA as a chemical crosslinker in the presence of AFP as a print molecule and PAAm-grafted lectins to form lectinAFP-antibody complexes. AFP-imprinted hydrogels were prepared by the removal of the print AFP from the resultant network, which was composed of lectin-AFP-antibody complexes. It is important to use a minute amount of crosslinker so that complex formation between ligand biomolecules and target AFP can induce structural changes in the hydrogel networks.

To investigate the effect of biomolecular imprinting on tumor marker-responsive behavior, the swelling ratio of AFP-imprinted hydrogels in the presence of AFP was compared with non-imprinted hydrogels prepared without AFP as a print. When the hydrogels were immersed in a phosphate buffer solution containing AFP, the AFPimprinted hydrogel began to shrink. However, non-imprinted hydrogel experienced only slight swelling. In addition, the swelling ratio of AFP-imprinted hydrogel depended on the concentration of AFP in the buffer solution. These results indicate that the AFP-imprinted hydrogel was tumor marker-responsive. Compressive modulus measurements showed that the crosslinking density of AFP-imprinted hydrogels increased gradually with increasing AFP concentration in the buffer solution, but the crosslinking density of the non-imprinted hydrogel did not change. Biomolecular imprinting enabled lectins and antibodies to be arranged at optimal positions for the simultaneous recognition of saccharide and peptide chains of AFP. Therefore, AFPresponsive shrinking of the AFP-imprinted hydrogel is due to the formation of sandwich-like lectin-AFP-antibody complexes that acted as reversible crosslinks.

a

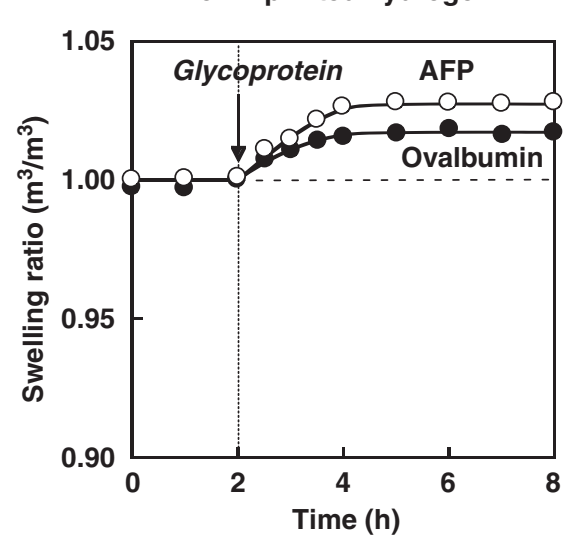

We also investigated the glycoprotein recognition behavior of AFPimprinted and non-imprinted hydrogels by measuring the change in the swelling ratio in response to AFP or ovalbumin (Figure 10). Ovalbumin possesses a saccharide chain similar to AFP but has a different peptide chain. Lectin used for the preparation of AFPimprinted and non-imprinted hydrogels can recognize the saccharide chain of ovalbumin but the antibody cannot recognize the peptide chain. Both AFP-imprinted and non-imprinted hydrogels swelled slightly in the presence of ovalbumin, which was similar to the behavior of non-imprinted hydrogels in the presence of AFP. Only AFP-imprinted hydrogels recognized AFP precisely and shrank in its presence. These results reveal that only AFP-imprinted hydrogels shrink gradually when both lectin and antibody ligands in the hydrogel simultaneously recognize saccharide and peptide chains of the target glycoprotein (Figure 9b). As a result, the swelling or shrinking behavior of AFP-imprinted hydrogels in the presence of glycoproteins enables the accurate detection and recognition of glycoproteins. Thus, biomolecular imprinting is a useful method for the preparation of biomolecule-responsive hydrogels with accurate detection and recognition of a target biomolecule, such as a tumorspecific marker. The fascinating responsive properties of biomoleculeimprinted hydrogels provide promising tools for the construction of novel sensor systems and molecular diagnostics with a wide variety of biomedical uses.

\section{STRUCTURAL EVALUATION OF HYDROGELS BY FRET}

Fluorescence resonance energy transfer (FRET) involves the transfer of excitation energy from a donor molecule to an acceptor molecule and takes place when the fluorescence emission band of the donor overlaps an excitation band of the acceptor. ${ }^{87}$ FRET has been used as a 'spectroscopic ruler' for measuring the distance between donor and acceptor molecules, which depend on the efficiency of FRET. For example, conformational changes of biomolecules, such as proteins and DNA labeled with a donor and an acceptor can be detected by measuring the fluorescence of the donor or acceptor molecule. ${ }^{8-90}$

The structure of cross-linked networks of hydrogels must be characterized because the structure directly affects the properties and function of the hydrogel. We proposed a method for the characterization of hydrogels using FRET, where an acceptor (quencher) was adsorbed on the polymer network bearing a fluores-

b

b AFP-imprinted hydrogel

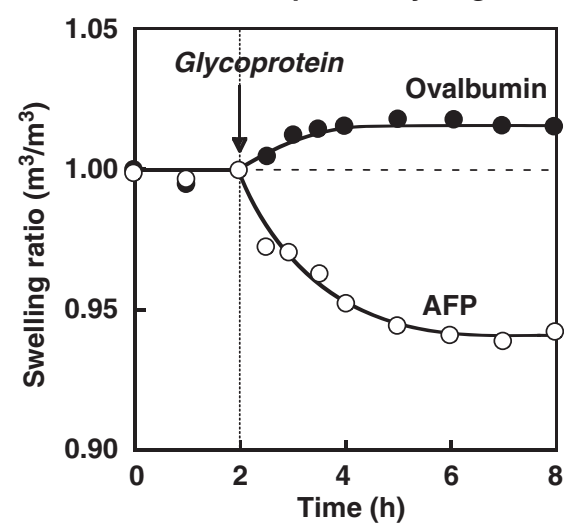

Figure 10 Changes in the swelling ratio of (a) non-imprinted and (b) AFP-imprinted hydrogels following the addition of AFP ( $O$ ) and ovalbumin (-) after swelling reached equilibrium in phosphate buffer solution at $25^{\circ} \mathrm{C}$. The glycoprotein concentration in the phosphate buffer solution was $40 \mu \mathrm{g} \mathrm{I}^{-1}$. Because ovalbumin possesses a saccharide chain similar to AFP and a peptide chain dissimilar to AFP, ovalbumin can be recognized by the lectin (ConA) but cannot be recognized by the antibody (antiAFP) in the hydrogels. 
cence donor (fluorophore) moiety. ${ }^{91}$ Furthermore, the measurement of fluorescence intensity changes induced by FRET revealed adsorption phenomena related to solutes in the polymer network on a nano-scale level. Acrylate monomer containing 5-(2'-aminoethyl)aminonaphthalene-1-sulfonic acid (EDANS), a common fluorophore, was synthesized by the coupling of EDANS with $N$-succinimidylacrylate and the resultant monomer was copolymerized with AAc and MBAA as a crosslinker. A fluorescence microscope image of the resultant PAAcEDANS hydrogel showed fluorescent emission from the EDANS moiety.

Changes in the fluorescence of PAAc-EDANS hydrogels by FRET were investigated using aminated 4-(4'-dimethylaminophenyl azo)benzoic acid (amino-DABCYL) as a cationic quencher, which was synthesized by coupling DABCYL with ethylenediamine and adsorbed on the anionic polymer network. Because the absorption spectrum of DABCYL overlaps the fluorescence emission spectrum of EDANS, ${ }^{92}$ FRET from EDANS to DABCYL occurred when the former was in the vicinity of the latter. The amino-DABCYL was adsorbed onto the PAAc-EDANS hydrogel by complex formation of negatively charged carboxyl or sulfonic acid groups in the PAAc-EDANS network with positively charged amino groups of amino-DABCYL. The fluorescence intensity of PAAc-EDANS hydrogels decreased markedly with an increase in the amount of amino-DABCYL adsorbed into the hydrogel.

The fluorescence intensity changes of PAAc-EDANS hydrogels were discussed according to the adsorption of amino-DABCYL using SternVolmer's equation (1), which represents the relationship between the concentration of the quencher and the fluorescence intensity when excitation is transferred from a fluorophore to a quencher by FRET. ${ }^{93}$

$$
\frac{F_{0}}{F}=1+K_{\mathrm{SV}}[Q]=1+k_{q} \tau_{0}[Q]
$$

where $F_{0}$ and $F$ are fluorescence intensities of a fluorophore in the absence and presence of a quencher, respectively, and $K_{\mathrm{SV}}$ is the Stern-Volmer's constant. The Stern-Volmer's plot showed that an approximately linear relationship existed between the amount of adsorbed amino-DABCYL and the relative fluorescence intensity of the PAAC-EDANS hydrogel (Figure 11). Therefore, we concluded that FRET occurred from EDANS to DABCYL in the PAAc-EDANS hydrogel.

Furthermore, using femtosecond laser spectroscopy, a useful tool for measuring fluorescence life time, we investigated fluorescence decay of EDANS in the PAAc-EDANS hydrogel by adsorption of amino-DABCYL. When EDANS in the PAAc-EDANS hydrogel was excited with UV light at $387.5 \mathrm{~nm}$, fluorescence emission was monitored at $480 \mathrm{~nm}$. Fluorescence from EDANS in the PAAc-EDANS hydrogel showed a more significant decay after the adsorption of amino-DABCYL when compared with the decay of fluorescence in the absence of amino-DABCYL. Furthermore, the fluorescence decay sharpened with increasing amounts of adsorbed amino-DABCYL. We determined the fluorescence lifetime of EDANS by fitting the curve of fluorescence decay using an exponential equation (2) consisting of two components. An equation consisting of one exponential component did not fit the fluorescence decay of the PAAc-EDANS hydrogel.

$$
\frac{I}{I_{0}}=a_{1} \exp \left(-\frac{t}{\tau_{1}}\right)+a_{2} \exp \left(-\frac{t}{\tau_{2}}\right)
$$

where $a_{1}$ and $a_{2}$ are pre-exponential constants and $\tau_{1}$ and $\tau_{2}$ are two types of fluorescence lifetimes, respectively. The fitting of the curve revealed that the PAAc-EDANS hydrogel and the hydrogel with adsorbed amino-DABCYL had two types of lifetimes for fluorescence decay. The mobility of EDANS in the PAAc-EDANS hydrogel showed
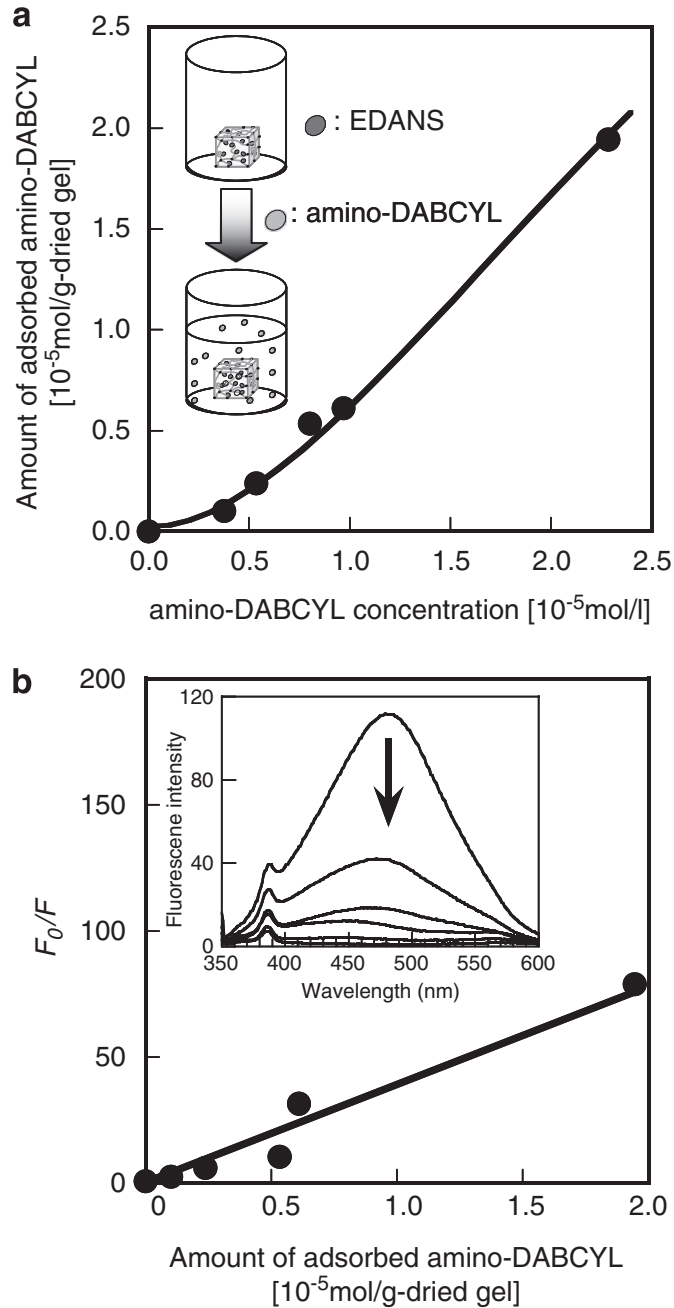

Figure 11 (a) Effect of the aqueous amino-DABCYL concentration on the amount of amino-DABCYL adsorbed into PAAC-EDANS hydrogels and (b) Stern-Volmer plot of PAAC-EDANS hydrogels with adsorbed amino-DABCYL. Inset: fluorescence spectrum of amino-DABCYL adsorbed PAAc-EDANS hydrogel. $\lambda_{\text {ex }}=340 \mathrm{~nm}$.

two modes. EDANS displayed high mobility when positioned far from crosslinks and low mobility in the vicinity of crosslinks (Figure 12). The fluorescence lifetimes of EDANS in the PAAc-EDANS hydrogel decreased upon adsorption of amino-DABCYL, suggesting that FRET occurred from EDANS to DABCYL, where EDANS acted as a fluorescence donor and DABCYL acted as an acceptor. In PAAcEDANS gels with a large amount of amino-DABCYL adsorbed, EDANS had a high probability of being in the vicinity of DABCYL and was able to quench its fluorescence. A short fluorescence life time was based on the high mobility of EDANS and decreased more strongly by the adsorption of amino-DABCYL than fluorescence with a long life time based on low mobility. Because FRET from high mobility EDANS to amino-DABCYL occurred more efficiently than from low mobility EDANS, the adsorption of amino-DABCYL caused a decrease in fluorescence with a short life time when compared with fluorescence with a long life time. The Förster radius $\left(\mathrm{R}_{0}\right)$ for FRET from EDANS to DABCYL is $3.3 \mathrm{~nm} .{ }^{94}$ Thus, the distance between EDANS in the PAAc-EDANS hydrogel and adsorbed amino-DABCYL is less than $3.3 \mathrm{~nm}$. The network structure of the 


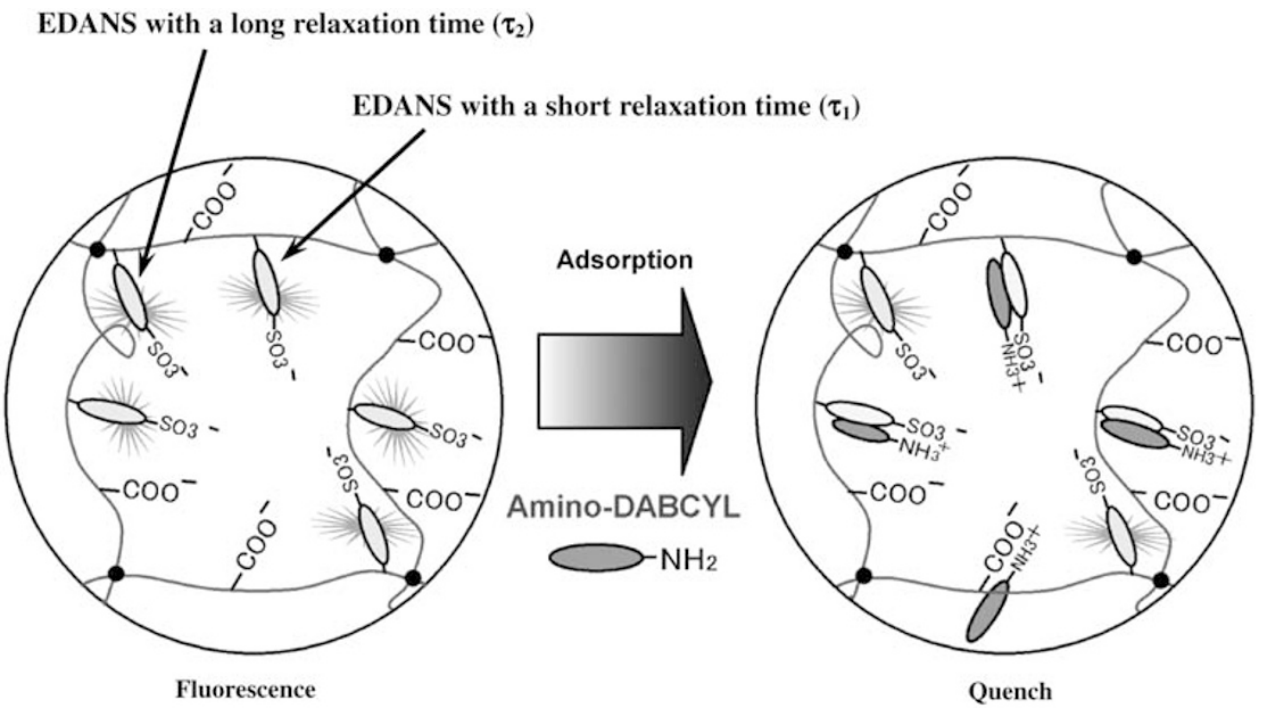

Figure 12 Illustration of FRET in PAAC-EDANS hydrogels.

hydrogel and the solute adsorption behavior evaluated by FRET was a result of complex formation between a negatively charged fluorophore and a positively charged acceptor.

\section{NETWORK DESIGN OF HYDROGELS}

The fascinating properties of stimuli-responsive hydrogels lead to many opportunities in the design of self-regulated drug delivery systems, actuators and so on. A rapid response is required for the use of stimuli-responsive hydrogels as smart materials. Based on Tanaka's theory, which suggests that the relaxation time for the swelling of a spherical hydrogel is proportional to the square of its radius and the inverse of the diffusion constant, ${ }^{95-97}$ the preparation of hydrogels that respond rapidly involves the control of physical structures including the size and shape of the hydrogel. ${ }^{98,99}$ On the other hand, the design of the chemical structure is another method for the creation of rapidly responsive hydrogels. For example, comb-type grafted hydrogels, in which the polymer chain has grafted side chains, can shrink rapidly in response to environmental changes. ${ }^{100-102}$ The rapid response of comb-type grafted hydrogels is caused by the inherent mobility of grafted chains bearing free ends.

To propose a new concept for the design of smart hydrogels that show responsive behavior, we focused on a semi-interpenetrating polymer network (semi-IPN) in which linear polymer chains with high mobility are entangled within cross-linked polymer networks. ${ }^{103}$ After linear PAAm was synthesized by polymerization of AAm, a PAAm hydrogel with semi-IPN structure was prepared by copolymerization of AAm and MBAA as a crosslinker in the presence of linear PAAm. The shrinkage response of the PAAm semi-IPN hydrogel in acetone/water mixtures was compared with a PAAm hydrogel consisting of only a cross-linked network without linear polymers (Figure 13). In spite of the same effect of acetone concentration on the equilibrium swelling ratio, a difference in shrinkage behavior between standard and semi-IPN hydrogels was observed. The standard hydrogel took more than $2 \mathrm{~h}$ to achieve equilibrium and the semi-IPN hydrogel shrank rapidly within $15 \mathrm{~min}$. This suggests that the introduction of a semi-IPN structure increases the shrinkage rate of a hydrogel.

The relaxation time for the shrinkage of standard and semi-IPN hydrogels was determined by curve fitting with equation (3). The

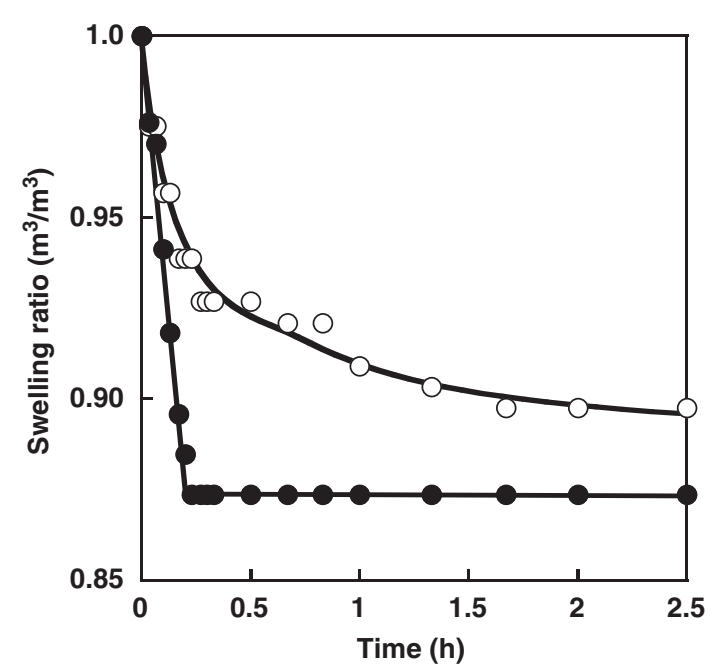

Figure 13 Changes in the swelling ratio of standard PAAm hydrogels $(O)$ and PAAm semi-IPN hydrogels $(\bullet)$ as a function of time after the hydrogels were pre-equilibrated in water and immersed into an acetone/water mixture with an acetone concentration of 10 vol $\%$ at $25^{\circ} \mathrm{C}$.

relaxation time is an indicator of the responsiveness of hydrogels governed by diffusion-limited transport of polymer chains.

$$
\text { Swelling ratio }=A_{0}+A_{1} \exp \left(-\frac{t}{\tau_{1}}\right)+A_{2} \exp \left(-\frac{t}{\tau_{2}}\right)
$$

where $A_{0}, A_{1}$ and $A_{2}$ are constants, and $\tau_{1}$ and $\tau_{2}$ are two types of relaxation for shrinkage. The relaxation time determined by the fitting of the curve revealed that the shrinkage of the hydrogels has two modes including short $\left(\tau_{1}\right)$ and long $\left(\tau_{2}\right)$ relaxation times. These relaxation times are assigned to the rapid shrinkage of free polymer chains distant from crosslinks and to the slow shrinkage of tied polymer chains close to crosslinks. The relaxation time of the semiIPN hydrogel during shrinking in an acetone/water mixture was much shorter than the standard hydrogel. A shorter relaxation time indicated that the semi-IPN hydrogel shrank faster than the standard 
a

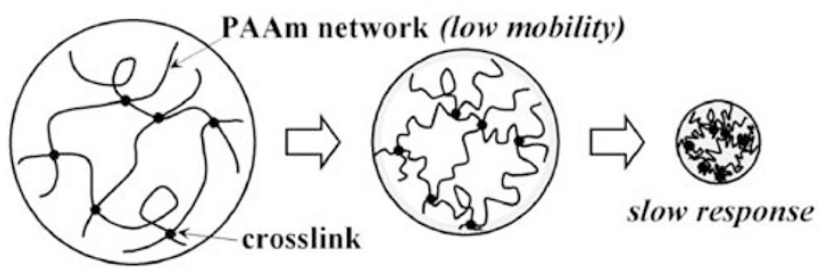

b

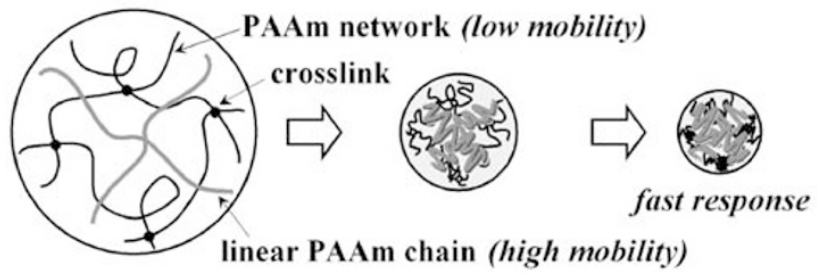

Figure 14 Tentative illustration of shrinkage of standard PAAm hydrogels (a) and PAAm semi-IPN hydrogels (b) in an acetone/water mixture.

hydrogel. As shown in Figure 14, the inherent mobility of linear polymer chains in the semi-IPN hydrogel can accelerate shrinkage of the cross-linked networks. Consequently, the design of network architecture by the introduction of a semi-IPN structure is of great importance for the enhancement of responsive behavior of a hydrogel.

\section{CONCLUSION}

This review describes our recent studies on the development of smart soft materials using molecular complexes. The use of molecular complexes derived from electrostatic interactions between a polymer network and a drug leads to the efficient loading of a charged drug within an oppositely charged hydrogel. The release of the drug was regulated in response to environmental changes. Using biomolecular complexes as reversible crosslinks in hydrogel networks enabled us to create biomolecule-responsive hydrogels that undergo a change in volume in response to a target biomolecule. In particular, we designed two kinds of biomolecule-responsive hydrogels including a biomolecule-crosslinked hydrogel that swelled in the presence of a target biomolecule and a biomolecule-imprinted hydrogel that shrank in the presence of the target. We also focused on network structures of smart hydrogels to improve stimuli-responsiveness. This review describes a method for characterizing network structure and adsorption behavior in smart hydrogels. FRET provides a useful tool for the characterization of network structure and solute adsorption behavior because the fluorescence intensity of hydrogels bearing fluorophores changes dramatically by the adsorption of a quencher. In addition, the introduction of a semi-IPN structure led to the rapid response of stimuli-responsive hydrogels due to the high mobility of linear polymer chains entangled in cross-linked polymer networks. The design of smart hydrogels using molecular complexes requires a detailed understanding of the structural factors that control performance and function. Knowledge gained from successful designs not only provides a basis for the development of smart soft materials with a wide variety of uses but also permits more insight into the fundamentals regarding the relationship between structure and function. In particular, the use of molecular complexes as crosslinks is a fascinating idea for the creation of stimuli-responsive hydrogels with molecular recognition functions. Despite the fact that most smart soft materials using molecular complexes still require further research into potential applications, they are likely to become important materials for the future.

\section{ACKNOWLEDGEMENTS}

The research achievements have been attained due to collaboration with many co-workers. We express our deep thanks to Professor T Uragami at Kansai University, Professor Emer K Nakamae and Professor T Nishino at Kobe University, Professor AS Hoffman at the University of Washington and Professor K Kato at Kyoto University for their helpful discussions. We are grateful to Dr K Okawa, Mr A Jikihara, Mrs Noriko Asami, Mr M Jige, Mr T Nakaminami, Mr T Nizuka and Mr M Furukawa for their cooperation in the experiments. This work was financially supported in part by The Ministry of Education, Culture, Sports, Science, and Technology (MEXT) of Japan, the Japan Society for the Promotion of Science (JSPS) and Japan Science and Technology Agency (JST).

1 Peppas, N. A. Hydrogels in Medicine and Pharmacy (CRC Press, Boca Raton, 1987).

2 DeRossi, D., Kajiwara, K., Osada, Y. \& Yamauchi, A. Polymer Gels, Fundamentals and Biomedical Applications (Plenum, New York, 1991).

3 Miyata, T. Gels and Interpenetrating Polymer Networks. Supramolecular Design for Biological Applications (ed. Yui, N.) Chapter 6, 95-136 (CRC Press, Boca Raton, 2002).

4 Dusek, K. Responsive Gels: Volume Transitions I, Adv. Polym. Sci. Vol. 109 (Springer, Berlin, 1993).

5 Dusek, K. Responsive Gels: Volume Transitions II, Adv. Polym. Sci. Vol. 110 (Springer, Berlin, 1993).

6 Okano, T. Biorelated Polymers and Gels (Academic Press, Boston, 1998).

7 Miyata, T. Stimuli-Responsive Polymers and Gels. Supramolecular Design for Biological Applications (ed. Yui, N.) Chapter 9, 191-225 (CRC Press, Boca Raton, 2002).

8 Tanaka, T., Fillmore, D., Sun, S.- T., Nishio, I., Swislow, G. \& Shah, A. Phase transitions in ionic gels. Phys. Rev. Lett. 45, 1636-1639 (1980).

9 Annaka, M. \& Tanaka, T. Multiple phases of polymer Gels. Nature 355, 430-432 (1992).

10 Tanaka, T. Collapse of gels and the critical endpoint. Phys. Rev. Lett. 40, 820-823 (1978).

11 Hirokawa, Y. \& Tanaka, T. Volume phase-transition in a nonionic gel. J. Chem. Phys. 81, 6379-6380 (1984).

12 Amiya, T., Hirokawa, Y., Hirose, Y., Li, Y. \& Tanaka, T. Reentrant phase-transition of $\mathrm{N}$-isopropylacrylamide gels in mixed-solvents. J. Chem. Phys. 86, 2375-2379 (1987).

13 Chen, G. \& Hoffman, A. S. Graft-copolymers that exhibit temperature-induced phasetransitions over a wide-range of $\mathrm{pH}$. Nature 373, 49-52 (1995).

14 Yoshida, R., Uchida, K., Kaneko, T., Sakai, K., Kikuchi, A., Sakurai, Y. \& Okano, T. Comb-type grafted hydrogels with rapid de-swelling response to temperature-changes. Nature 374, 240-242 (1995).

15 Tanaka, T., Nishio, I., Sun, S.- T. \& Ueno-Nishio, S. Collapse of gels in an electric field. Science 218, 467-469 (1982).

16 Osada, Y., Okuzaki, H. \& Hori, H. A polymer gel with electrically driven motility. Nature 355, 242-244 (1992).

17 Irie, M. Stimuli-responsive poly(N-Isopropylacrylamide)—photoinduced and chemical-induced phase-transitions. Adv. Polym. Sci. 110, 49-65 (1993).

18 Suzuki, A. \& Tanaka, T. Phase-transition in polymer gels induced by visible-light. Nature 346, 345-347 (1990).

19 Siegel, R. A. Hydrophobic weak polyelectrolyte gels-studies of swelling equilibria and kinetics. Adv. Polym. Sci. 109, 233-267 (1993).

20 Brannon-Peppas, L. \& Peppas, N. A. Solute and penetrant diffusion in swellable polymers. 9. The mechanisms of drug release from $\mathrm{pH}$-sensitive swelling-controlled systems. J. Controlled Release 8, 267-274 (1989).

21 Brazel, C. S. \& Peppas, N. A. Synthesis and characterization of thermomechanically and chemomechanically responsive poly ( $\mathrm{N}$-isopropylacrylamide-co-methacrylic acid) hydrogels. Macromolecules 28, 8016-8020 (1995).

22 Dong, L.- C. \& Hoffman, A. S. A novel-approach for preparation of $\mathrm{pH}$-sensitive hydrogels for enteric drug delivery. J. Controlled Release 15, 141-152 (1991).

23 Hoffman, A. S. Applications of thermally reversible polymers and hydrogels in therapeutics and diagnostics. J. Controlled Release 6, 297-305 (1987).

24 Okano, T. Molecular design of temperature-responsive polymers as intelligent materials. Adv. Polym. Sci. 110, 179-197 (1993).

25 Dong, L.- C. \& Hoffman, A. S. Synthesis and application of thermally reversible heterogels for drug delivery. J. Controlled Release 13, 21-31 (1990).

26 Okano, T., Bae, Y. H., Jacobs, H. S. \& Kim, W. Thermally on off switching polymers for drug permeation and release. J. Controlled Release 11, 255-265 (1990).

27 Katono, H., Maruyama, A., Sanui, K., Ogata, N., Okano, T. \& Sakurai, Y. Thermoresponsive swelling and drug release switching of interpenetrating polymer networks composed of poly (acrylamide-co-butyl methacrylate) and poly (acrylic-acid). J. Controlled Release 16, 215-227 (1991). 
28 Miyata, T., Uragami, T. \& Nakamae, K. Biomolecule-sensitive hydrogels. Adv. Drug Delivery Rev. 54, 79-98 (2002).

29 Miyata, T. \& Uragami, T. Biological stimuli-responsive hydrogels. Polymeric Biomaterials (ed. Dumitriu, S.) Chapter 36, 959-974 (Marcel Dekker Inc., New York, 2002).

30 Miyata, T. Biomolecule-Responsive Gels: Design of Smart Gels Using Biomolecular Interactions, Bottom-Up Nanofabrication: Supramolecules, Self-Assemblies, and Organized Films (eds. Ariga, K., Nalwa, H. S.) Volume 6, Chapter 9, 137-150 (American Scientific Publishers, Los Angeles, 2009).

31 Ishihara, K., Kobayashi, M., Ishimaru, N. \& Shinohara, I. Glucose-induced permeation control of insulin through a complex membrane consisting of immobilized glucoseoxidase and a poly(amine). Polym. J. 16, 625-631 (1984).

32 Ishihara, K. \& Matsui, K. Glucose-responsive insulin release from polymer capsule. J. Polym. Sci.: Polym. Lett. Ed. 24, 413-417 (1986).

33 Albin, G., Horbett, T. A. \& Ratner, B. D. Glucose sensitive membranes for controlled delivery of insulin: Insulin transport studies. J. Controlled Release 2, 153-164 (1985)

34 Cartier, S., Horbett, T. A. \& Ratner, B. D. Glucose-sensitive membrane coated porous filters for control of hydraulic permeability and insulin delivery from a pressurized reservoir. J. Membrane Sci. 106, 17-24 (1995).

35 Hassan, C. M., Doyle, F. J. III \& Peppas, N. A. Dynamic behavior of glucose-responsive poly(methacrylic acid-g-ethylene glycol) hydrogels. Macrmolecules 30, 6166-6173 (1997).

36 Parker, R. S., Doyle, F. J. III \& Peppas, N. A. A model-based algorithm for blood glucose control in type I diabetic patients. IEEE Trans. Biomed. Eng. 46, 148-157 (1999).

37 Kataoka, K., Miyazaki, H., Okano, T. \& Sakurai, Y. Sensitive glucose-induced change of the lower critical solution temperature of poly [N, N-dimethylacrylamide-co-3(acrylamido) phenyl-boronic acid] in physiological saline. Macromolecules 27, 1061-1062 (1994)

38 Kataoka, K., Miyazaki, H., Bunya, M., Okano, T. \& Sakurai, Y. Totally synthetic polymer gels responding to external glucose concentration: their preparation and application to on-off regulation of insulin release. J. Am. Chem. Soc. 120, 12694 12695 (1998).

39 Matsumoto, A., Kurata, T., Shiino, D. \& Kataoka, K. Swelling and shrinking kinetics of totally synthetic, glucose-responsive polymer gel bearing phenylborate derivative as a glucose-sensing moiety. Macromolecules 37, 1502-1510 (2004).

40 Matsumoto, A., Yoshida, R. \& Kataoka, K. Glucose-responsive polymer gel bearing phenylborate derivative as a glucose-sensing moiety operating at the physiological $\mathrm{pH}$. Biomacromolecules 5, 1038-1045 (2004).

41 Miyata, T., Nakamae, K., Hoffman, A. S. \& Kanzaki, Y. Stimuli-sensitivities of hydrogels containing phosphate groups. Macromol. Chem. Phys. 195, 1111-1120 (1994).

42 Nakamae, K., Nizuka, T., Miyata, T., Furukawa, M., Nishino, T., Kato, K., Inoue, T., Hoffman, A. S. \& Kanzaki, Y. Lysozyme loading and release from hydrogels carrying pendant phosphate groups. J. Biomater. Sci., Polymer Edn. 9, 43-53 (1997).

43 Nakamae, K., Nishino, T., Kato, K., Miyata, T. \& Hoffman, A. S. Synthesis and characterization of stimuli-sensitive hydrogels having a different length of ethylene glycol chains carrying phosphate groups: loading and release of lysozyme. J. Biomaterials Sci., Polym. Ed. 15, 1435-1446 (2004).

44 Dong, L. C. \& Hoffman, A. S. A novel-approach for preparation of $\mathrm{pH}$-sensitive hydrogels for enteric drug delivery. J. Controlled Release 15, 141-152 (1991).

45 Hoffman, A. S., Stayton, P. S., Bulmus, V., Chen, G., Chen, J., Cheung, C., Chilkoti, A., Ding, Z., Dong, L., Fong, R., Lackey, C. A., Long, C. J., Miura, M., Morris, J. E., Murthy, N., Nabeshima, Y., Park, T. G., Press, O. W., Shimoboji, T., Shoemaker, S., Yang, H. J., Monji, N., Nowinski, R. C., Cole, C. A., Priest, J. H., Harris, J. M., Nakamae, K., Nishino, T. \& Miyata, T. Really smart bioconjugates of smart polymers and receptor proteins. J. Biomed. Mater. Res. 52, 577-586 (2000).

46 Brownlee, M. \& Cerami, A. A glucose-controlled insulin-delivery system: semisynthetic insulin bound to lectin. Science 206, 1190-1191 (1979).

47 Seminoff, L. A., Olsen, G. B. \& Kim, S. W. A self-regulating insulin delivery system. 1. characterization of a synthetic glycosylated insulin derivative. In. J. Pharm. 54, 241-249 (1989)

48 Kim, S. W., Pai, C. M., Makino, K., Seminoff, L. A., Holmberg, D. L., Gleeson, J. M., Wilson, D. E. \& Mack, E. J. Self-regulated glycosylated insulin delivery. J. Control. Release 11, 193-201 (1990)

49 Makino, K., Mack, E. J., Okano, T. S. \& Kim, W. A microcapsule self-regulating delivery system for insulin. J. Control. Release 12, 235-239 (1990).

50 Obaidat, A. A. \& Park, K. Characterization of glucose dependent gel-sol phase transition of the polymeric glucose-concanavalin A hydrogel system. Pharm. Res. 13, 989-995 (1996)

51 Obaidat, A. A. \& Park, K. Characterization of protein release through glucose-sensitive hydrogel membranes. Biomaterials 18, 801-806 (1997).

52 Kokufuta, E., Zhang, Y.- Q. \& Tanaka, T. Saccharide-sensitive phase-transition of a lectin-loaded gel. Nature 351, 302-304 (1991).

53 Miyata, T. \& Nakamae, K. Polymers with pendant saccharides -'Glycopolymers'. Trends in Polymer Science 5, 198-206 (1997).

54 Nakamae, K. Miyata, T. \& Ootsuki, N. Evaluation of surface characteristics of polymers in water -Measurement of surface free energy in water-. Makromol. Chem., Rapid. Commun. 14, 413-420 (1993).

55 Nakamae, K., Miyata, T., Ootsuki, N., Okumura, M. \& Kinomura, K. Surface characterizations of copolymer films with pendant monosaccharides. Macromol. Chem. Phys. 195, 1953-1963 (1994).
56 Nakamae, K., Miyata, T., Ootsuki, N., Okumura, M. \& Kinomura, K. Surface studies on copolymers having pendant monosaccharides. Macromol. Chem. Phys. 195, 26632675 (1994).

57 Miyata, T., Ootsuki, N., Nakamae, K., Okumura, M. \& Kinomura, K. Protein adsorption on a copolymer having pendant monosaccharide groups-relationship between surface free energy and protein adsorption. Macromol. Chem. Phys. 195, 3597-3607 (1994).

58 Miyata, T., Morizane, M., Nakamae, K., Okumura, M. \& Kinomura, K. Properties of biomedical pressure-sensitive adhesive copolymer films with pendant monosaccharides. J. Appl. Polym. Sci. 56, 1615-1623 (1995).

59 Nakamae, K., Miyata, T., Jikihara, A. \& Hoffman, A. S. Formation of poly(glucosyloxyethyl methacrylate)-concanavalin A complex and its glucose-sensitivity. J. Biomaterials Sci., Polym. Ed. 6, 79-90 (1994).

60 Miyata, T., Jikihara, A., Nakamae, K. \& Hoffman, A. S. Preparation of poly(glucosyloxyethyl methacrylate)-concanavalin A complex hydrogel and its glucose-sensitivity. Macromol. Chem. Phys. 197, 1135-1146 (1996).

61 Miyata, T., Jikihara, A., Nakamae, K. \& Hoffman, A. S. Preparation of reversibly glucose-responsive hydrogels by covalent immobilization of lectin in polymer networks having pendant glucose. J. Biomaterials Sci., Polym. Ed. 15, 1085-1098 (2004).

62 Ulijn, R. V. Enzyme-responsive materials: a new class of smart biomaterials. J. Mater. Chem. 16, 2217-2225 (2006)

63 Yeh, P.- Y., Kopeckova, P. \& Kopecek, J. Biodegradable and pH-sensitive hydrogelssynthesis by cross-linking of $\mathrm{N}, \mathrm{N}$-dimethylacrylamide copolymer precursors. J. Polym. Sci.: Part A; Polym. Chem. 32, 1627-1637 (1994).

64 Plunkett, K. N., Berkowski, K. L. \& Moore, J. S. Chymotrypsin responsive hydrogel: application of a disulfide exchange protocol for the preparation of methacrylamide containing peptides. Biomacromolecules 6, 632-637 (2005).

65 Li, C., Madsen, J., Armes, S. P. \& Lewis, A. L. A new class of biochemically degradable, stimulus-responsive triblock copolymer gelators. Angew. Chem. Int. Ed. 45, 3510-3513 (2006)

66 Yamamoto, N., Kurisawa, M. \& Yui, N. Double-stimuli-responsive degradable hydrogels: interpenetrating polymer networks consisting of gelatin and dextran with different phase separation. Macromol. Rapid. Commun. 17, 313-318 (1996)

67 Huh, K. M., Hashi, J., Ooya, T. \& Yui, N. Synthesis and characterization of dextran grafted with poly(N-isopropylacrylamide-co-N,N-dimethyl-acrylamide). Macromol. Chem. Phys. 201, 613-619 (2000).

68 Sperinde, J. J. \& Griffith, L. G. Synthesis and characterization of enzymatically-crosslinked poly(ethylene glycol) hydrogels. Macromolecules 30, 5255-5264 (1997).

$69 \mathrm{Hu}$, B.- H. \& Messersmith, P. B. Rational design of transglutaminase substrate peptides for rapid enzymatic formation of hydrogels. J. Am. Chem. Soc. 125, 14298-14299 (2003).

70 Thornton, D. P., McConnell, G. \& Ulijn, R. V. Enzyme responsive polymer hydrogel beads. Chem. Commun. 47, 5913-5915 (2005).

71 Thornton, P. D., Mart, R. J. \& Ulijn, R. V. Enzyme-responsive polymer hydrogel particles for controlled release. Adv. Mater. 19, 1252-1256 (2007).

72 Diamandis, E. P. \& Christopoulos, T. K. Immunoassay (Academic Press, New York, 1996)

73 Miyata, T., Asami, N. \& Uragami, T. A reversibly antigen-responsive hydrogel. Nature 399, 766-769 (1999).

74 Miyata, T., Asami, N. \& Uragami, T. Preparation of an antigen-sensitive hydrogel using antigen-antibody bindings. Macromolecules 32, 2082-2084 (1999).

75 Miyata, T., Asami, N. \& Uragami, T. Structural design of stimuli-responsive bioconjugated hydrogels that respond to a target antigen. J. Polym. Sci., Polym. Phys. 47, 2144-2157 (2009)

76 Wulff, G., Sarhan, A. \& Zabrocki, K. Enzyme-analog built polymers and their use for the resolution of racemates. Tetrahedron Lett. 44, 4329-4332 (1973).

77 Sellergren, B., Lepisto, M. \& Mosbach, K. Highly enantioselective and substrateselective polymers obtained by molecular imprinting utilizing noncovalent interaction-NMR and chromatographic studies on the nature of recognition. J. Am. Chem. Soc. 110, 5853-5860 (1988).

78 Mosbach, K. Molecular imprinting. Trends Biochem. Sci. 19, 9-14 (1994).

79 Shea, K. J. Molecular imprinting of synthetic network polymers: the de novo synthesis of macromolecular binding and catalytic sites. Trends Polym. Sci. 2, 166-173 (1994).

80 Wulff, G. Molecular imprinting in cross-linlinked mterials with the aid of molecuar templates- a way towards artificial antibodies. Angew. Chem., Int. Ed. Engl. 34, 1812-1832 (1995)

81 Byrne, M., Park, K. \& Peppas, N. A. Molecular imprinting within hydrogels. Adv. Drug Deliv. Rev. 54, 149-161 (2002)

82 Bergmann, N. M. \& Peppas, N. A. Molecularly imprinted polymers with specific recognition for macromolecules and proteins. Prog. Polym. Sci. 33, 271-288 (2008)

83 Watanabe, M., Akahoshi, T., Tabata, Y. \& Nakayama, D. Molecular specific swelling change of hydrogels in accordance with the concentration of guest molecules. J. Am. Chem. Soc. 120, 5577-5578 (1998)

84 Oya, T., Enoki, T., Grosberg, A. Y., Masamune, S., Sakiyama, T., Takeoka, Y., Tanaka, K., Wang, G. Q., Yilmaz, Y., Feld, M. S., Dasari, R. \& Tanaka, T. Reversible molecular adsorption based on multiple-point interaction by shrinkable gels. Science 286, 1543-1545 (1999)

85 Miyata, T., Jige, M., Nakaminami, T. \& Uragami, T. Tumor marker-responsive behavior of gels prepared by biomolecular imprinting. Proc. Natl. Acad. Sci. USA 103, 11901193 (2006) 
86 Aoyagi, Y., Suzuki, Y., Isemura, M., Nomoto, M., Sekine, C., Igarashi, K. \& Ichida, F. The fucosylation index of alpha-fetoprotein and its usefulness in the early diagnosis of hepatocellular carcinoma. Cancer 61, 769-774 (1988).

87 Stryer, L. Fluorescence energy transfer as a spectroscopic ruler. Ann. Rev. Biochem. 47, 819-846 (1978).

88 Tyagi, S. \& Kramer, F. R. Molecular beacons: probes that fluoresce upon hybridization. Nat. Biotechnol. 14, 303-308 (1996).

89 Tyagi, S., Bratu, D. P. \& Kramer, F. R. Multicolor molecular beacons for allele discrimination. Nat. Biotechnol. 16, 49-53 (1998).

90 Fang, X., Liu, X., Schuster, S. \& Tan, W. Designing a novel molecular beacon for surface-immobilized DNA hybridization studies. J. Am. Chem. Soc. 121, 2921-2922 (1999).

91 Okawa, K., Miyata, T. \& Uragami, T. Fluorescence resonance energy transfer by quencher adsorption into hydrogels containing fluorophore. J. Polym. Sci.: Part B Polym. Phys. 44, 3245-3252 (2006).

92 Haugland, R. P. in Handbook of Fluorescent Probes and Research Products, 9th edn (ed. Gregory, J.) 432 (Molecular Probes, Inc., Eugene, 2002).

93 Bell, J. H., Schairer, E. T., Hand, L. A. \& Mehta, R. D. Surface pressure measurements using luminescent coatings. Annu. Rev. Fluid Mech. 33, 155-206 (2001).

94 Haugland, R. P. in Handbook of Fluorescent Probes and Research Products, 9th edn (ed. Gregory, J.) p 25 (Molecular Probes, Inc., Eugene, 2002).

95 Tanaka, T. \& Filmore, D. J. Kinetics of swelling of gels. J. Chem. Phys. 70, 1214-1218 (1979).
96 Tanaka, T., Sato, E., Hirokawa, Y., Hirotsu, S. \& Peetermans, J. Critical kinetics of volume phase transition of gels. Phys. Rev. Lett. 55, 2455-2458 (1985).

97 Shibayama, M. \& Tanaka, T. Volume phase transition and related phenomena of polymer gels. Adv. Polym. Sci. 109, 1-62 (1993).

98 Kabra, B. G. \& Gehrke, S. H. Synthesis of fast-response, temperature-sensitive poly(Nisopropylacrylamide) gel. Polym. Commun. 32, 322-323 (1991).

99 Wu, X. S., Hoffman, A. S. \& Yager, P. Synthesis and characterization of thermally reversible macroporous poly(N-isopropylacrylamide) hydrogels. J. Polym. Sci., A. Polym. Chem. 30, 2121-2129 (1992).

100 Yoshida, R., Uchida, K., Kaneko, Y., Sakai, K., Kikuchi, A., Sakurai, Y. \& Okano, T. Comb-type grafted hydrogels with rapid de-swelling response to temperature changes. Nature 374, 240-242 (1995).

101 Kaneko, Y., Sakai, K., Kikuchi, A., Yoshida, R., Sakurai, Y. \& Okano, T. Influence of freely mobile grafted chain length on dynamic properties of comb-type grafted poly $(\mathrm{N}$ isopropylacrylamide) hydrogels. Macromolecules 28, 7717-7723 (1995).

102 Kaneko, Y., Nakamura, S., Sakai, K., Aoyagi, T., Kikuchi, A., Sakurai, Y. \& Okano, T. Rapid deswelling response of poly( $\mathrm{N}$-isopropylacrylamide) hydrogels by the formation of water release channels using poly(ethylene oxide) graft chains. Macromolecules 31, 6099-6105 (1998).

103 Miyata, T., Asami, N., Okawa, K. \& Uragami, T. Rapid response of a poly(acrylamide) hydrogels having semi-interpenetrating polymer network (semi-IPN) structure. Polym. Adv. Tech. 17, 794-797 (2006).

Takashi Miyata is currently a Professor of Chemistry and Materials Engineering at Kansai University. He was born in Osaka, Japan in 1964. He earned a B. Eng. Degree (1987) and M. Eng. Degree (1989) from Kobe University under the supervision of Professor Katsuhiko Nakamae. He quitted the Ph.D. course and moved to Kansai University as an Assistant Professor in 1991. He received a D. Eng. Degree from Kobe University in 1994. He was promoted to an Associate Professor (1999) and to a full Professor (2008) at Kansai University. He was concurrently appointed as Researcher of PRESTO (2002-2006, Conversion and Control by Advanced Chemistry: 2006-Present, Structures and Control of Interfaces) of Japan Science and Technology Agency (JST). He received the Young Investigator Award of The Membrane Society of Japan (2002), the Young Investigator Award of The Adhesion Society of Japan (2003) and Wiley Award from Society of Polymer Science, Japan (2007). His research interests include functional polymer science, particularly polymer gels, polymer membranes and polymer surface chemistry. 